\title{
An ovine CFTR variant as a putative cystic fibrosis causing mutation
}

\author{
Scott J Tebbutt, Ann Harris, Diana F Hill
}

\begin{abstract}
This report describes a DNA variant in the ovine cystic fibrosis transmembrane conductance regulator (CFTR) gene that has been previously reported as a putative cystic fibrosis causing mutation in humans. The variant is a guanine to adenine base change at position 1019 of the ovine CFTR cDNA, corresponding to an arginine (R) to glutamine $(Q)$ amino acid substitution at position 297 in the predicted CFTR polypeptide. The equivalent R297Q mutation in exon 7 of the human CFTR gene has been reported in a CF patient. This is the first putative cystic fibrosis mutation to be detected in another animal species.
\end{abstract}

( $($ Med Genet 1996;33:623-624)

Key words: cystic fibrosis; CFTR mutations; animal homologues.

Cystic fibrosis (CF) is associated with mutations in the cystic fibrosis transmembrane conductance regulator (CFTR) gene, ${ }^{1}$ which encodes a small conductance chloride ion channel, ${ }^{2}$ and may have other additional functions as a regulator of other ion channels. ${ }^{3}$ Extensive analysis of the CFTR gene in patients and carriers has shown over 600 DNA changes, including point, frameshift, and splice site mutations (CF Genetic Analysis Consortium, unpublished data). However, only a few mutations
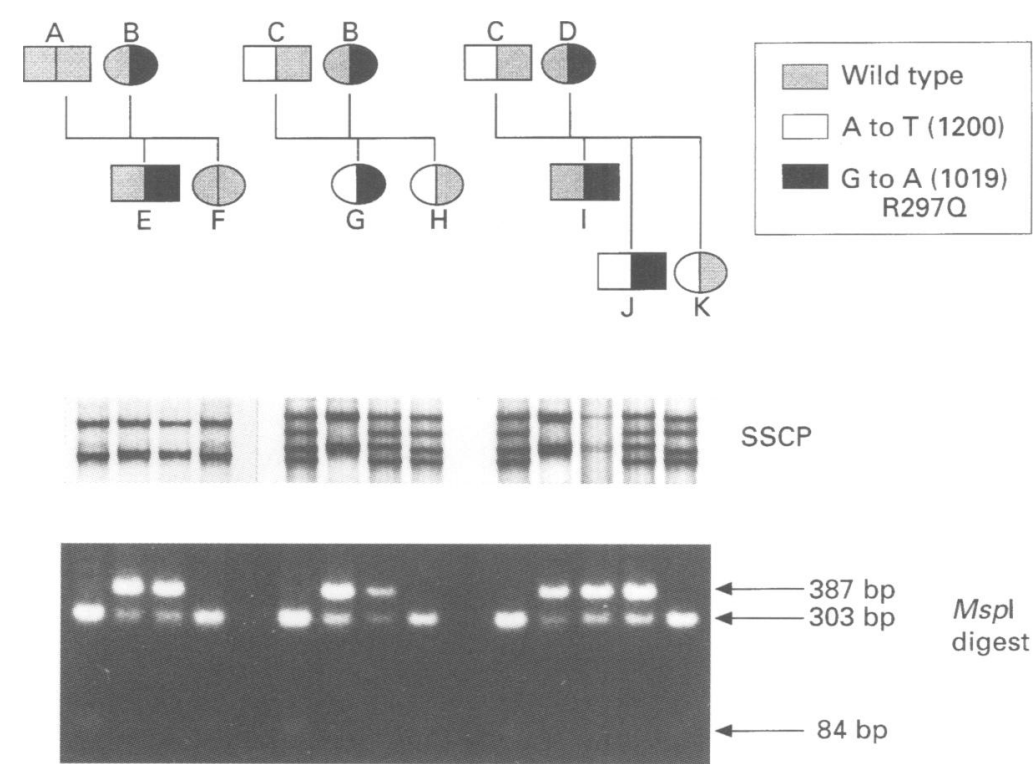

Mspl digest

Figure 1 Pedigree structure of sheep carrying the G1019A (R297Q) CFTR exon 7 gene variant in the heterozygote state (also a silent polymorphism, A1200T, unpublished data). SSCP analysis of exon 7 is shown, as well as the MspI restriction analysis. have been shown to influence CFTR $\mathrm{Cl}^{-}$channel activity, and much work is still required to understand how specific mutations cause disease.

No natural animal models of CF have been identified, although several lines of transgenic CF mice have been generated that reflect the pathology of the disease to a greater or lesser extent. ${ }^{4}$ Recently, more advanced techniques of homologous recombination and mouse embryonic stem cell manipulation have resulted in the generation of transgenic CF mice carrying the $\Delta$ F508 CFTR mutation, the most frequent $C F$ associated mutation. ${ }^{5}$ Nevertheless, CF mice models have limitations and there is still a need for a natural animal model to help study the pathology of CF lung disease and for evaluating pharmacological approaches to $\mathrm{CF}$ treatment. We are investigating sheep as a potential model for $\mathrm{CF}^{6}$ Genetic screening for polymorphisms of ovine CFTR in natural sheep populations has identified several DNA sequence changes. Here, we describe the identification of one such DNA variant that has a putative CF causing equivalent in humans.

Single stranded conformation polymorphism (SSCP) analysis was carried out on ovine genomic DNA, using primers specific for ovine CFTR intron sequences either side of the exon (ov7i5: GGAAAGTATATAAGCACC and ov7i3: AGAGAGTTTGCTCATGAC) which corresponds to the human exon 7 sequence. Methods were based on those of Shackleton et al. ${ }^{7}$ SSCP mobility shifts were detected in several sheep DNA samples (fig 1), and one SSCP was found to be the result of a guanine to adenine substitution at base pair position 1019 in the ovine CFTR cDNA sequence. ${ }^{6}$ This substitution destroys a $M s p$ I site, resulting in a $387 \mathrm{bp}$ fragment instead of the two component fragments (303 and $84 \mathrm{bp}$ ) observed when the site is present. MspI restriction analysis on amplified exon 7 DNA from sheep families carrying the variant confirmed Mendelian inheritance (fig 1). Ewe "G" is heterozygous for the R297Q variant.

To confirm the expression of this mutation, reverse transcriptase (RT) PCR was performed on blood lymphocyte mRNA from ewe "G". A specific fragment of the ovine CFTR cDNA was amplified using the method of Chalkley and Harris. ${ }^{8}$ The two primer sets were as follows: first set $5^{\prime}$ AST2R: GCAGATGAGAATAGCTATG 3' (and RT primer) BST1L: CTCCATCACTACATCTG; second (nested) set 5' AST3R: GATGAAGGACTTGCACTGG 3' A2LA: CAATGCAGAATGAGATGG. MspI restriction analysis on the purified PCR product was consistent with the 


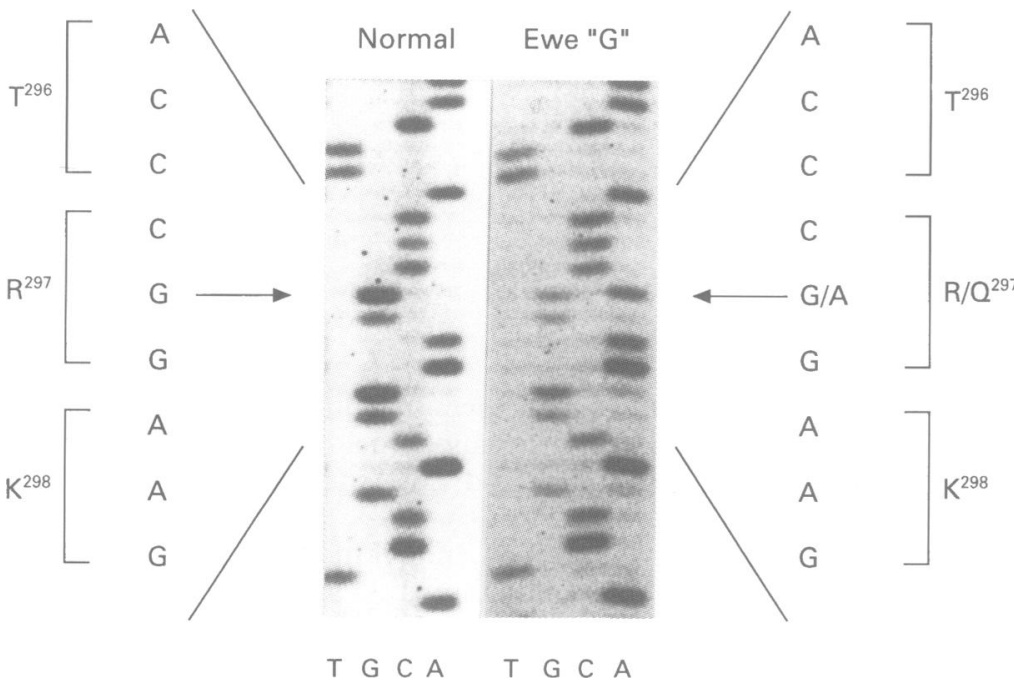

Figure 2 Sequence analysis of exon 7 of the CFTR gene transcripts from "normal" and a G1019A carrying sheep (ewe " $G$ "). RT-PCR products were directly sequenced. Ewe " $G$ " is heterozygous for a $G$ to $A$ base change at ovine CFTR cDNA position 1019, resulting in a predicted arginine to glutamine $(R$ to $Q)$ amino acid substitution.

exon 7 variant being an expressed allele (data not shown). DNA sequence analysis was used to confirm that the $\mathrm{R} 297 \mathrm{Q}$ allele is efficiently transcribed (fig 2).

The ovine DNA variant reported here ( $\mathrm{G}$ to A at position 1019) causes an arginine (R) to glutamine $(\mathrm{Q})$ change at codon 297 in the predicted CFTR polypeptide. This is homologous to a putative cystic fibrosis causing mutation ( $R 297 Q)$ reported in a Northern Ireland family. ${ }^{9}$ Interestingly, the arginine (R297) residue lies in the first membrane spanning domain of the predicted CFTR protein, specifically part of the cytoplasmic loop between the putative transmembrane helices 4 and $5 .{ }^{1}$ The threonine-arginine-lysine (TRK) peptide in this region is entirely conserved in the predicted polypeptides from all animal species in which the CFTR gene has been characterised, ${ }^{10}$ including human, sheep, cattle, mouse, Xenopus, and dogfish. This seems consistent with a non-conservative amino acid change at R297 eliciting a functional change in the CFTR protein, perhaps leading to a cystic fibrosis phenotype.

The two human CF patients reported to carry the R297Q mutation were aged 6 and 8 years (in 1991). Clinically, both patients were similarly affected with mild to moderate chest symptoms and both were receiving pancreatic enzyme supplements. ${ }^{9}$ A recent study of a French CF family ${ }^{11}$ has suggested that R297Q represents a rare polymorphism, rather than a disease causing mutation, or that the length of a $T$ tract in intron 8 may play a role in influencing the severity of the R297Q allele. ${ }^{12} \mathrm{We}$ are currently investigating any equivalent $T$ tract length variation in intron 8 of the ovine CFTR gene. At present, it is uncertain if $\mathrm{R} 297 \mathrm{Q}$ is a disease causing mutation. Nevertheless, if R297Q in the human CFTR protein does indeed contribute to the cystic fibrosis phenotype, it is tempting to consider that another animal species, carrying R297Q in the homozygous state, might also show some of the symptoms of CF. The ewe "G", who is heterozygous for R297Q, seems perfectly healthy. We are establishing a breeding programme to obtain lambs that are homozygous for the R297Q variant.

We would like to thank Hannah Henry for her help in providing retrospective sheep DNA samples, and Ann Beattie for her assistance in obtaining fresh blood samples from the appropriate sheep. This work was supported in part by the AgResearch Molecular Biology Unit (University of Otago), the Medical Research Council (UK), the EPA Research Trust, and the Cystic Fibrosis Research Trust (UK). SJT is the recipient of a Cystic Fibrosis Research Trust (UK). SJT is
University of Otago Postdoctoral Fellowship.

1 Riordan J, Rommens J, Kerem BS, et al. Identification of the cystic fibrosis gene: cloning and characterization of complementary DNA. Science 1989;245:1066-73.

2 Anderson M, Rich D, Gregory R, Smith A, Welsh $M$ Generation of cAMP-activated chloride currents by expression of CFTR. Science 1991;251:679-82.

3 Schwiebert E, Egan M, Hwang TH, et al. CFTR regulates outwardly rectifying chloride channels through an autooutwardly rectifying chloride channels through an auto-
crine mechanism involving ATP. Cell 1995;81:1063-73.

4 Dorin J, Dickinson P, Alton E, et al. Cystic fibrosis in the mouse by targeted insertional mutagenesis. Nature 1992; mouse by targe

5 Colledge W, Abella B, Southern K, et al. Generation and characterization of a $\triangle \mathrm{F} 508$ cystic fibrosis mouse model. Nature Genet 1995;10:445-52.

6 Tebbutt S, Wardle C, Hill D, Harris A. Molecular analysis of the ovine cystic fibrosis transmembrane conductance regulator gene. Proc Natl Acad Sci USA 1995;92:2293-7.

7 Shackleton S, Hull J, Dear S, Seller A, Thomson A, Harris A. Identification of rare and novel mutations in the CFTR genes of CF patients in southern England. Hum Muta 1994;3:141-51.

8 Chalkley G, Harris A. Lymphocyte mRNA as a resource for detection of mutations and polymorphisms in the CF gene. $\mathcal{f}$ Med Genet 1991;28:777-80.

9 Graham C, Goon P, Hill A, Cutting G, Curristan S, Nevin $\mathrm{N}$. Identification of a new mutation (R297O) in exon 7 of the CFTR gene in a Northern Ireland family. $\mathcal{J}$ Med of the CFTR gene
Genet 1991;28:571.

10 Tucker S, Tannahill D, Higgins C. Identification and developmental expression of the Xenopus laevis cystic fibrosis transmembrane conductance regulator gene. Hum Mol Genet 1992;1:77-82.

11 Dorval I, Jezequel P, Chauvel B, et al. French CF family genotype analysis shows that the $\mathrm{R} 297 \mathrm{Q}$ mutation is a rare polymorphism. Hum Mutat 1995;6:334-5.

12 Dorval I, Jezequel P, Roussey M, et al. A French CF family genetic analysis shows that the R297Q associated with the T7 allele in the intron 8 polypyrimidine track is not involved in the CF disease. Pediatr Pulmonol 1994;10:216 (abst 132). 